\title{
Regional Homogeneity Brain Alterations in Schizophrenia: An Activation Likelihood Estimation Meta-Analysis
}

\author{
Xiaolei Qiu ${ }^{1 *}$, Wenwen $\mathrm{Xu}^{2 \star}$, Rongrong Zhang ${ }^{1 *}$, Wei Yan ${ }^{1}$, Wenying $\mathrm{Ma}^{2}$, Shiping $\mathrm{Xie}^{1 凶}$, and Min Zhou ${ }^{1 凶}$ \\ ${ }^{1}$ Department of Psychiatry, the Affiliated Brain Hospital of Nanjing Medical University, Nanjing, China \\ ${ }^{2}$ Department of Neurology, the Affiliated Brain Hospital of Nanjing Medical University, Nanjing, China
}

\begin{abstract}
Objective Resting state functional magnetic resonance imaging (rsfMRI) provides a lot of evidence for local abnormal brain activity in schizophrenia, but the results are not consistent. Our aim is to find out the consistent abnormal brain regions of the patients with schizophrenia by using regional homogeneity (ReHo), and indirectly understand the degree of brain damage of the patients with drug-naive first episode schizophrenia (Dn-FES) and chronic schizophrenia.
\end{abstract}

Methods We performed the experiment by activation likelihood estimation (ALE) software to analysis the differences between people with schizophrenia group (all schizophrenia group and chronic schizophrenia group) and healthy controls.

Results Thirteen functional imaging studies were included in quantitative meta-analysis. All schizophrenia group showed decreased ReHo in bilateral precentral gyrus (PreCG) and left middle occipital gyrus (MOG), and increased ReHo in bilateral superior frontal gyrus (SFG) and right insula. Chronic schizophrenia group showed decreased ReHo in bilateral MOG, right fusiform gyrus, left PreCG, left cerebellum, right precuneus, left medial frontal gyrus and left anterior cingulate cortex (ACC). No significant increased brain areas were found in patients with chronic schizophrenia.

Conclusion Our findings suggest that patients with chronic schizophrenia have more extensive brain damage than FES, which may contribute to our understanding of the progressive pathophysiology of schizophrenia.

Psychiatry Investig 2021;18(8):709-717

Key Words Schizophrenia, Regional homogeneity, Resting state, Meta-analysis, ALE.

\section{INTRODUCTION}

Schizophrenia characterized by disturbances in various aspects such as perceptual, cognitive, social, emotional, and linguistic is a sort of severe mental disorder with high recurrence rate, and disability rate. ${ }^{1}$ About $0.3 \%-0.7 \%$ schizophrenia patients worldwide are diagnosed according to epidemiological data. ${ }^{2}$ Some studies showed that schizophrenia is actually a typical brain connection disorder, resulting in hallucination and delusion, which is considered as typical positive psychotic

Received: February 16, 2021 Revised: April 26, 2021

Accepted: May 24, 2021

$\triangle$ Correspondence: Shiping Xie, MD, PhD

Department of Psychiatry, the Affiliated Brain Hospital of Nanjing Medical University, No. 264, Guangzhou Road, Gulou District, Nanjing 210029, China Tel: +86-13851588810, Fax: +86-025-82296224, E-mail: xieshiping6365@126.com

$\triangle$ Correspondence: Min Zhou, MD, PhD

Department of Psychiatry, the Affiliated Brain Hospital of Nanjing Medical University, No. 264, Guangzhou Road, Gulou District, Nanjing 210029, China Tel: +86-13913988826, Fax: +86-025-82296361, E-mail: zhou.cn@163.com

*These authors contributed equally to this work.

(a) This is an Open Access article distributed under the terms of the Creative Commons Attribution Non-Commercial License (https://creativecommons.org/licenses/bync/4.0) which permits unrestricted non-commercial use, distribution, and reproduction in any medium, provided the original work is properly cited. symptoms of schizophrenia. ${ }^{3}$ However, the pathophysiological mechanism of classification of schizophrenia has still not been clarified. ${ }^{4}$

In recent years, an increasing number of neuroimaging studies have focused on the use of resting state functional magnetic resonance imaging (rsfMRI) to explore the pathophysiology of neurodevelopment in schizophrenia. ${ }^{5}$ Regional homogeneity (ReHo), a highly reliable rsfMRI measurement method, measures the similarity between a given voxel and its nearest BOLD signal time series in a voxel way through Kendall's coefficient of concordance (KCC), providing important information about time synchronization of brain regions. ${ }^{6,7}$ Evidence indicates that the underlying pathophysiological mechanism of schizophrenia may be associated with several brain neural networks, including default network (DMN), visual network (VN) and salience network (SN). ${ }^{8}$ However, the consequences of schizophrenia focused on ReHo study are inconsistent. For example, some studies have reported increased ReHo in frontal lobe in schizophrenia, ${ }^{9,10}$ however, others have shown the opposite results. ${ }^{11,12}$ Moreover, the abnormal brain regions identified in these local consistency studies were multiple. A rs- 
fMRI meta-analysis confirmed that the brain dysfunction in each stage were distinct. ${ }^{4}$ As is known to all, schizophrenia falls into two categories: drug-naive first episode schizophrenia (Dn-FES) and chronic schizophrenia. The difference between the two categories mainly lies in the course of disease and the influence of drugs, in addition, compared with Dn-FES, patients with chronic schizophrenia experience more social stress and stressors and indicated more extensive brain damage. Therefore, aggregating the eligible studies to achieve consistent results makes a lot of sense for identifying the respective neurobiological markers, which needs to be done by effective means of meta-analysis. Activation likelihood estimation (ALE), a coordinate-based meta-analysis, reveals image-specific markers by avoiding laboratory bias in the most consistent spatial changes of brain structure and function across studies. ${ }^{13}$

To our knowledge, a few meta-analysis of rsfMRI studies have revealed local neural connectivity abnormalities in schizophrenia. Kuhn et al. ${ }^{14}$ performed a meta-analysis on the resting brain activity of schizophrenia and depression patients. Xiao et al. ${ }^{15}$ conducted a meta-analysis of local abnormal connections in schizophrenia and their unaffected relatives. Nevertheless, existing studies did not detailly clarify the difference between Dn-FES and chronic schizophrenia, possibly because of the unavailability of data. As a result, it is necessary to use ALE algorithm to make a quantitative meta-analysis of the consistent brain alterations on patients with schizophrenia classified according to their course of illness.

Hence, the purpose of the present study was to conduct two group comparsions: 1 ) all schizophrenia (Dn-FES and chronic schizophrenia) and healthy controls (HCs); 2) chronic schizophrenia and HCs. It was hypothesized that: the findings of all schizophrenia patients and chronic patients would show the special ReHo abnormalities which belong to the current known network.

\section{METHODS}

\section{Literature selection}

The meta-analysis of neuroimaging studies was conducted according to the PRISMA statement and recorded using the suggested checklist.

\section{Search strategy}

We have systematically searched all articles in Pubmed, Embase, Web of science and Cochrane Library. The search strategy was performed with Medical Subject Heading (MESH) keywords and their related phrases in "Schizophrenia” [MeSH] OR "Schizophrenias" [Title/Abstract] OR "Schizophrenic disorders" [Title/Abstract]) OR "disorder schizophrenic" [Title/Abstract] OR "disorders schizophren- ic" [Title/Abstract]) OR "Schizophrenic Disorder" [Title/Abstract] OR "Dementia Praecox" [Title/Abstract] or "schizophr*" [Title/Abstract]; AND "ReHo" [Title/Abstract] OR "Regional homogeneity" [Title/Abstract]) OR "local consistency" [Title/Abstract] OR "coherence" [Title/Abstract]; AND "functional magnetic resonance imaging" [Title/Abstract] OR "fMRI" [Title/Abstract]) OR "resting state" [Title/ Abstract] (Supplementary Table 1 in the online-only Data Supplement).

\section{Inclusion and exclusion criteria}

Studies will be included if the article met the following conditions: 1) the research target had been diagnosed with schizophrenia; 2) studies were rsfMRI study, use of ReHo; 3) studies based on whole-brain analysis; 4) studies were compared with patients with schizophrenia and HCs group; 5) studies were reported cluster information (e.g. coordinates, cluster size, thresholding approaches); 6) studies were published on English language journal.

Studies will be excluded if the article met the following conditions: 1) studies were not imaging study; 2) without peer review; 3) not whole brain study (e.g. region of interest, volume of interest, support vector machine); 4) articles were not provided coordinate data or even after contacting the authors by email remained unavailable; 5) studies were not original study (e.g. letters to the editor, review articles, case reports, and animal experimental studies ); 6) studies were not published in English journals; 7) cohe ReHo (coherent regional homogeneity) was used instead of KCC ReHo; 8) unable to obtain full text.

\section{Data extraction and quality evaluation}

We extract useful data from the selected studies as follows: author, years of publication, gender distribution, sample size, diagnosis and classification system of schizophrenia, diagnosis, age (mean $\pm \mathrm{SD}$ ), scanner (magnetic field strength), eye state, repetition time/echo time, thickness, slice gap, full-width at half-maximum (FWHM, threshold), and drug states, furthermore, we collected $\mathrm{x}, \mathrm{y}$, and $\mathrm{z}$ coordinates reported using Talairach or Montreal Neurological Institute (MNI), which have statistically significant findings. If necessary, information is missing, we will contact the author to obtain original article. We used a 10-point checklist that included specific methods for clinical and demographic and imaging to assess the quality of the literature included basic information and suitable studies (Supplementary Table 2 in the online-only Data Supplement). Literature search, study selection and data collection were performed independently by two psychiatrists (W.Y.M. and W.W.X.), and any differences were judged by two experienced psychiatrists (W.Y. and R.R.Z.). 


\section{Statistical analysis}

The data analysis was dealt with GingerALE software (www.brainmap.org). ${ }^{16}$ ALE is a widely used coordinated based meta-analysis method that regards the focus of the report as a spital distribution centered on a given coordinate. ${ }^{17,18}$ ALE technology regards the focus of the report as a spatial probability distribution centered on a given coordinate, rather than a spatial probability distribution focused on a point. ${ }^{19}$ This method allows the creation of an activated probability map using the focus of different articles and compares it with a random spatial distribution, and the analysis carried out in MNI space. ${ }^{20}$ In order to assure statistical conspicuousness, we used 1,000 simulations to test arrangement of randomly distributed focuses, the cluster level Family Wise Error (FWE) of ALE map was $p<0.001$ and the cluster-forming threshold was $p<0.001 .^{21}$ To illustrate this, We use the neuroimaging and brain network visualization software BrainNet Viewer (http://www.nitrc.org/projects/bnv/) to visualize the brain maps of ALE. ${ }^{21}$

We first conducted ALE meta-analysis on all schizophrenia patients, then excluded four diagnoses as the Dn-FES (the duration of the disease was less than 2 years) patients experiments (because the research on the first episode schizophrenia patients are relatively small, the significance of combination is not significant, so this study did not combine the first episode patients) and a partial drug-naïve study, after that, conducted meta-analysis on the chronic schizophrenia patients (the duration of the disease was less than 2 years).

\section{RESULTS}

\section{Included studies and sample features}

Through literature search of four databases (pubmed, web of science, embase, cochrane library), we have retrieved 445 documents. After initially removing the duplicates and browsing the title and summary, we found that 28 studies met the

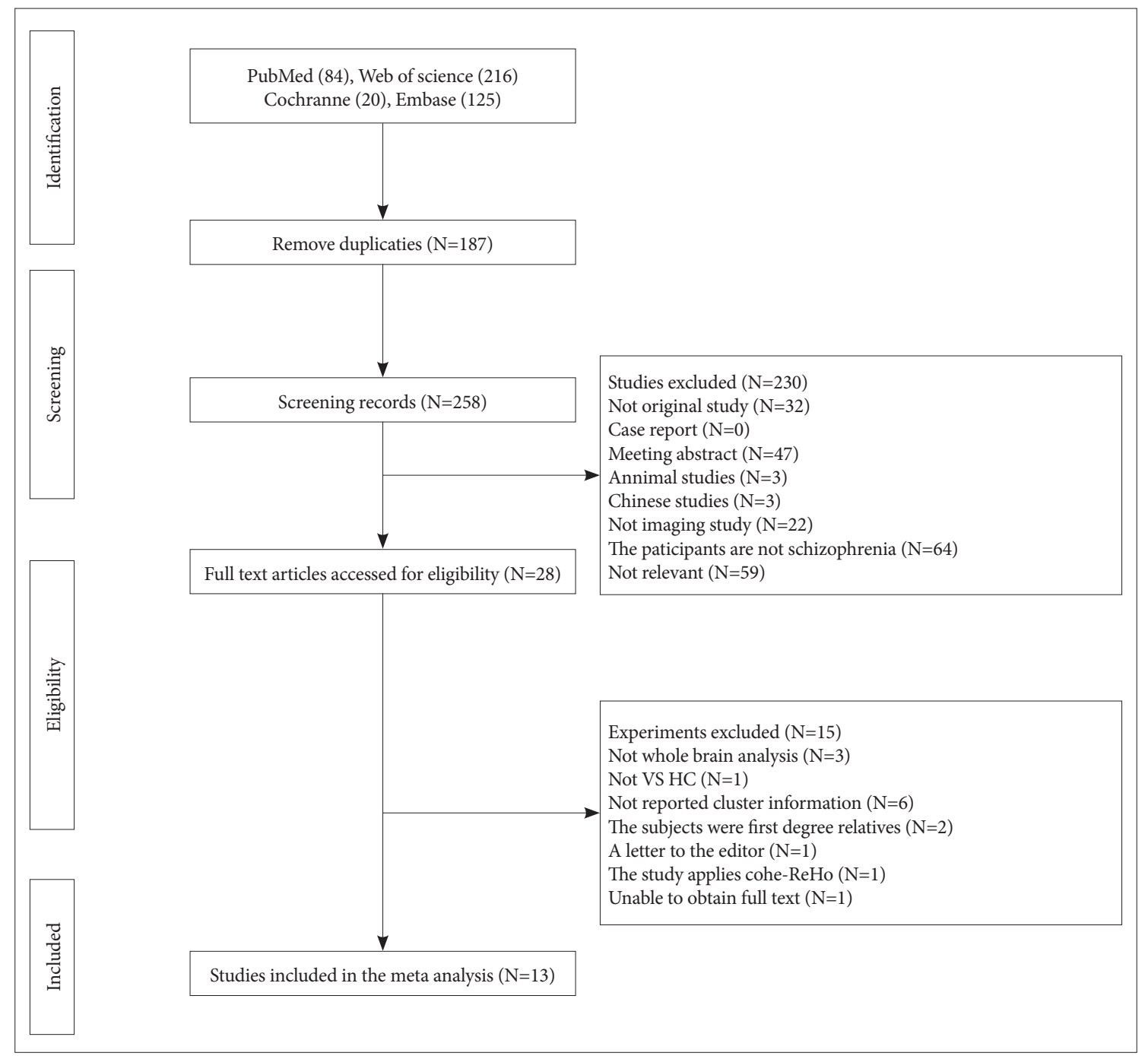

Figure 1. Flow-diagram of studies selection. 
inclusion criteria, furthermore, after a detailed review of the full text, a total of 13 studies met the inclusion criteria. ${ }^{8,9,11,12,22-30}$ Figure 1 illustrates the identification and exclusion flow chart for the study. The included research reported ReHo differences in 511 schizophrenia (193 with FES and 318 with chronic schizophrenia), and compared with 452 HCs. The research characteristics and quality scores included in this meta-analysis are presented in Tables 1 and 2.

\section{ReHo brain alterations comparison between all schizophrenia and HCs}

The comparison of ALE meta-analysis results between all schizophrenia group and HCs group are briefly summarized in Figure 2 and Table 3. The comparison between all schizophrenia and healthy control group showed decreased ReHo in bilateral precentral gyrus (PreCG) and left middle occipital gyrus (MOG), whereas increased ReHo in bilateral superior frontal gyrus (SFG) and right insula (Table 3, Figure 2).

\section{ReHo brain alterations comparison between chronic schizophrenia and HCs}

The comparison of ALE meta-analysis results between all schizophrenia group and HCs group are summarized in Figure 3 and Table 4. We found that decreased ReHo in bilateral MOG, right fusiform gyrus (FFG), left PreCG, left cerebellum, right precuneus (PCUN), left medial frontal gyrus and left anterior cingulate (ACC) (Table 4, Figure 3). No significant abnormal increased brain areas were found in the chronic schizophrenia.

\section{DISCUSSION}

Our study is the first quantitative meta-analysis, which combines the whole brain ReHo results of rsfMRI study in different stages of schizophrenia in a voxel way. Our advantage lies in the systematic and comprehensive understanding of schizophrenia in three groups (all schizophrenia group, chronic group, HCs group).

Table 1. Demographic data and clinical information of included study

\begin{tabular}{|c|c|c|c|c|c|c|c|c|}
\hline Author & $\begin{array}{l}\text { Sample } \\
\text { size }\end{array}$ & $\begin{array}{c}\text { Sex } \\
(\mathrm{M} / \mathrm{F})\end{array}$ & $\begin{array}{c}\text { Diagnostic } \\
\text { criteria }\end{array}$ & $\begin{array}{c}\text { SZ age } \\
(\text { mean } \pm S D)\end{array}$ & $\begin{array}{c}\text { HC age } \\
(\text { mean } \pm S D)\end{array}$ & Diagnosis & Drug state & $\begin{array}{c}\text { Quanlity } \\
\text { score }\end{array}$ \\
\hline Shan et al. ${ }^{22}$ & $\begin{array}{l}\text { SZ } 39 \\
\text { HC } 20\end{array}$ & $\begin{array}{c}27 / 12 \\
14 / 6\end{array}$ & DSM-5 & $24.64 \pm 5.01$ & $25.7 \pm 4.9$ & $\mathrm{SZ}$ & Yes & 9.5 \\
\hline Zhao et al. ${ }^{23}$ & $\begin{array}{l}\text { SZ } 44 \\
\text { HC } 26\end{array}$ & $\begin{array}{c}31 / 13 \\
17 / 9\end{array}$ & DSM-IV & $23.7 \pm 5.3$ & $22.6 \pm 3.7$ & FES & Drug naive & 10 \\
\hline Zhang et al. ${ }^{24}$ & $\begin{array}{l}\text { SZ } 53 \\
\text { HC } 67\end{array}$ & $\begin{array}{l}42 / 11 \\
46 / 21\end{array}$ & DSM-IV & $36.7 \pm 13.67$ & $34.82 \pm 11.28$ & SZ & Yes & 9.5 \\
\hline Wang et al. ${ }^{8}$ & $\begin{array}{l}\mathrm{SZ} 48 \\
\mathrm{HC} 31\end{array}$ & $\begin{array}{l}21 / 27 \\
14 / 17\end{array}$ & DSM-IV-TR & $15.79 \pm 1.64$ & $15.42 \pm 1.52$ & FES & Drug naive & 10 \\
\hline Gou et al. ${ }^{25}$ & $\begin{array}{l}\mathrm{SZ} 28 \\
\mathrm{HC} 21\end{array}$ & $\begin{array}{c}16 / 12 \\
14 / 7\end{array}$ & DSM-IV & $23.9 \pm 5.4$ & $28.8 \pm 6.1$ & SZ & Yes & 9.5 \\
\hline Gao et al. ${ }^{27}$ & $\begin{array}{l}\text { SZ } 34 \\
\text { HC } 29\end{array}$ & $\begin{array}{l}19 / 15 \\
16 / 13\end{array}$ & DSM-IV & $35.13 \pm 8.85$ & $32.73 \pm 7.61$ & SZ & Yes & 10 \\
\hline Liu et al. ${ }^{11}$ & $\begin{array}{l}\text { SZ } 27 \\
\text { HC } 27\end{array}$ & $\begin{array}{c}15 / 12 \\
18 / 9\end{array}$ & DSM-IV & $25.44 \pm 5.92$ & $27.44 \pm 7.24$ & $\mathrm{SZ}$ & Partly & 9.5 \\
\hline Cui et al. ${ }^{28}$ & $\begin{array}{l}\text { SZ } 32 \\
\mathrm{HC} 19\end{array}$ & NA & DSM-IV-TR & NA & NA & FES & Drug naive & 9 \\
\hline Gao et al. ${ }^{26}$ & $\begin{array}{l}\text { SZ } 14 \\
\mathrm{HC} 14\end{array}$ & $\begin{array}{l}9 / 5 \\
9 / 5\end{array}$ & DSM-IV & $33.2 \pm 10.7$ & $34.9 \pm 113.6$ & SZ & Yes & 9.5 \\
\hline Yu et al. ${ }^{9}$ & $\begin{array}{l}\text { SZ } 69 \\
\text { HC } 62\end{array}$ & $\begin{array}{l}34 / 35 \\
25 / 27\end{array}$ & DSM-IV & $31.7 \pm 9.6$ & $29.9 \pm 8.6$ & SZ & Yes & 9.5 \\
\hline Liu et al. ${ }^{12}$ & $\begin{array}{l}\text { SZ } 18 \\
\text { HC } 18\end{array}$ & $\begin{array}{l}9 / 9 \\
9 / 9\end{array}$ & DSM-IV & $23.67 \pm 4.397$ & $24.44 \pm 3.884$ & SZ & Yes & 9 \\
\hline Chen et al. ${ }^{30}$ & $\begin{array}{l}\text { SZ } 36 \\
\text { HC } 44\end{array}$ & $\begin{array}{l}16 / 20 \\
17 / 27\end{array}$ & DSM-IV & $28.28 \pm 1.43$ & $35.68 \pm 1.81$ & SZ & Yes & 9 \\
\hline Yan et al. ${ }^{29}$ & $\begin{array}{l}\text { SZ } 69 \\
\text { HC } 74\end{array}$ & $\begin{array}{l}50 / 19 \\
45 / 29\end{array}$ & DSM-IV & $24.22 \pm 7.08$ & $26.27 \pm 6.97$ & FES & Drug naive & 10 \\
\hline
\end{tabular}

SZ: schizophrenia, FES: first-episode schizophrenia, HC: healty control, NA: not available, SD: standard deviation, M: male, F: female, DSM: Diagnostic and Statistical Manual of Mental Disorders 
Table 2. Imaging information of the included studies

\begin{tabular}{|c|c|c|c|c|c|c|c|c|}
\hline Author & Scanners & Eye state & $\begin{array}{c}\text { Repetition time/ } \\
\text { echo time }\end{array}$ & $\begin{array}{c}\text { Thickness } \\
(\mathrm{mm})\end{array}$ & $\begin{array}{c}\text { Slice gap } \\
(\mathrm{mm})\end{array}$ & $\begin{array}{c}\text { FWHM } \\
(\mathrm{mm})\end{array}$ & Threshold & Coordinate \\
\hline Shan et al. ${ }^{22}$ & $3.0 \mathrm{~T}$ & Closed & $2,000 / 30 \mathrm{~ms}$ & 4 & 0.6 & 4 & Corrected & MNI \\
\hline Zhao et al. ${ }^{23}$ & $3.0 \mathrm{~T}$ & Closed & $2,000 / 30 \mathrm{~ms}$ & 4 & 0.5 & 4 & Corrected & MNI \\
\hline Zhang et al. ${ }^{24}$ & $3.0 \mathrm{~T}$ & Closed & $2,000 / 29 \mathrm{~ms}$ & 3 & NA & 6 & Corrected & MNI \\
\hline Wang et al. ${ }^{8}$ & $3.0 \mathrm{~T}$ & NA & $2,000 / 30 \mathrm{~ms}$ & 4 & 0.6 & 4 & Corrected & MNI \\
\hline Gou et al. ${ }^{25}$ & $1.5 \mathrm{~T}$ & Closed & $2,000 / 40 \mathrm{~ms}$ & 5 & 1 & 4 & Corrected & MNI \\
\hline Gao et al. ${ }^{27}$ & $3.0 \mathrm{~T}$ & NA & $2,000 / 30 \mathrm{~ms}$ & 4 & 0.6 & 4 & Corrected & MNI \\
\hline Liu et al. ${ }^{11}$ & $1.5 \mathrm{~T}$ & NA & $2,000 / 40 \mathrm{~ms}$ & 5 & 1 & 8 & Uncorrected & MNI \\
\hline Cui et al. ${ }^{28}$ & $3.0 \mathrm{~T}$ & Closed & $2,000 / 30 \mathrm{~ms}$ & 4 & 0.6 & 4 & Corrected & MNI \\
\hline Gao et al. ${ }^{26}$ & $1.5 \mathrm{~T}$ & Closed & $2,000 / 40 \mathrm{~ms}$ & 5 & 1 & 6 & Corrected & MNI \\
\hline Yu et al. ${ }^{9}$ & $3.0 \mathrm{~T}$ & NA & $2,000 / 24 \mathrm{~ms}$ & 3 & 0 & 6 & Corrected & MNI \\
\hline Liu et al. ${ }^{12}$ & $1.5 \mathrm{~T}$ & NA & $2,000 / 40 \mathrm{~ms}$ & 5 & 1 & 4 & Corrected & MNI \\
\hline Chen et al. ${ }^{30}$ & $3.0 \mathrm{~T}$ & Closed & $580 / 18 \mathrm{~ms}$ & 4 & 0 & 4 & Corrected & Talairach \\
\hline Yan et al. ${ }^{29}$ & $3.0 \mathrm{~T}$ & Closed & $2,500 / 30 \mathrm{~ms}$ & 3.5 & 1 & 4 & Corrected & MNI \\
\hline
\end{tabular}

FWHM: full width at half maximum, MNI: montreal neurological institute
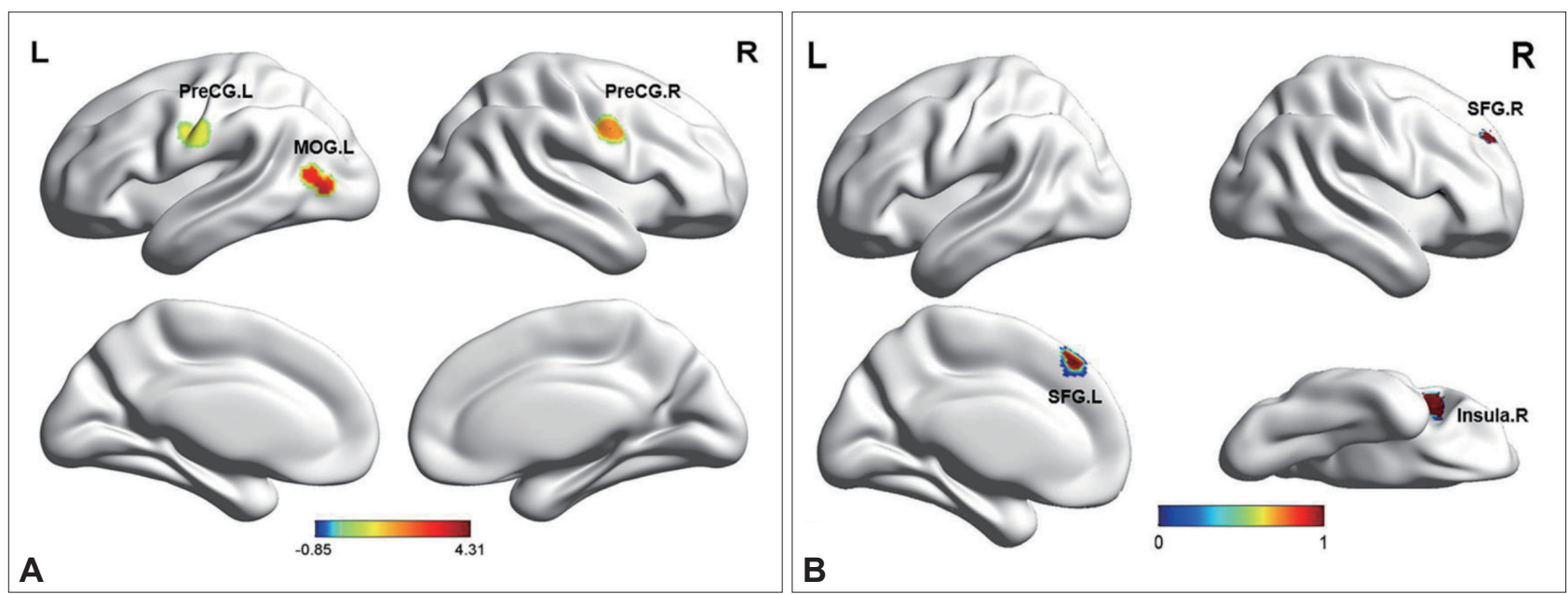

Figure 2. A: Decreased ReHo in all schizophrenia patients compared with healthy controls. B: Increased ReHo in all schizophrenia patients compared with healthy controls. PreCG: precentral gyrus, MOG: middle occipital gyrus, R: right, L: left, SFG: superior frontal gyrus.

Table 3. Altered ReHo in all schizophrenia patients compared with healthy controls

\begin{tabular}{|c|c|c|c|c|c|c|}
\hline Cluster & Cluster size $\left(\mathrm{mm}^{3}\right)$ & Local extrema $(\mathrm{x}, \mathrm{y}, \mathrm{z})$ & Location & Side & ALE value & Brodman \\
\hline \multicolumn{7}{|c|}{ Decreased ReHo } \\
\hline 1 & 2,264 & $-60,-10,34$ & PreCG & $\mathrm{L}$ & 0.002365635 & 4 \\
\hline 2 & 1,656 & $54,-2,34$ & PreCG & $\mathrm{R}$ & 0.002120542 & 6 \\
\hline 3 & 1,072 & $-44,-68,8$ & MOG & $\mathrm{L}$ & 0.0018646559 & 37 \\
\hline 3 & 1,072 & $-44,-74,4$ & MOG & $\mathrm{L}$ & 0.0017760438 & 37 \\
\hline \multicolumn{7}{|c|}{ Increased ReHo } \\
\hline 1 & 2,840 & $0,36,48$ & SFG & $\mathrm{L}$ & 0.0019955738 & 8 \\
\hline 2 & 928 & $42,20,10$ & Insula & $\mathrm{R}$ & 0.0015351484 & 13 \\
\hline 3 & 336 & $24,48,38$ & SFG & $\mathrm{R}$ & 0.0014410246 & 8 \\
\hline
\end{tabular}

PreCG: precentral gyrus, MOG: middle occipital gyrus, SFG: superior frontal gyrus, R: right, L: left 


\section{The Dn-FES in comparison with chronic patients indirectly}

As assumed, both the two groups had regional abnormal of varying degrees. The significant finding of our meta-analysis is that there are obvious alterations in frontal and occipital regions in all schizophrenia. Meanwhile, in chronic schizophrenia, there are more extensive local consistent changes, involving frontal, temporal, parietal, and occipital regions and even cerebellum. Compare with all schizophrenia group, the results showed that both the Dn-FES and chronic schizophrenia suffered from the common damage of occipital lobe and frontal lobe, which were not affected by the course of disease and medication. It is suggested that these brain regions may be abnormal in the early stage of schizophrenia, as well as not affected by the occurrence and development of disease.

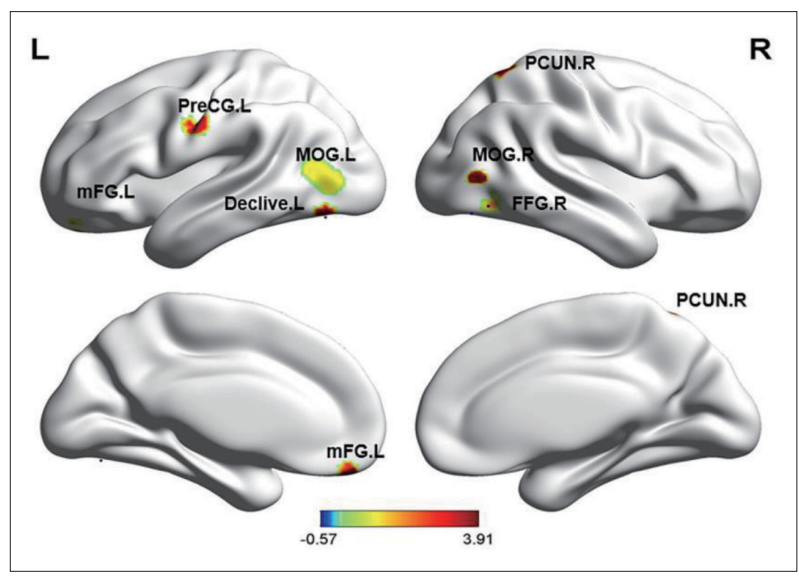

Figure 3. Decreased ReHo in chronic schizophrenia patients compared with healthy controls. MOG: middle occipital gyrus, FFG: fusiform gyrus, PCUN: precuneus, mFG: medial frontal gyrus, ACC: anterior cingulate cortex, R: right, L: left.

\section{Specific imaging abnormal markers}

Our meta-analysis found consistent areas of decreased ReHo in the MOG in all schizophrenia patients compared with HCs and chronic schizophrenia patients compared with HCs. Occipital lobe lesions that cause hallucinations, color blindness, or agraphia have been confirmed. ${ }^{31}$ As the core area of the VN, MOG is mainly responsible for processing visual function. ${ }^{31}$ In schizophrenia, structural and functional abnormalities of VN have been previously reported. ${ }^{32}$ For example, the association between visual hallucinations and $\mathrm{VN}$ damage in schizophrenia patients has been confirmed, and the total volume of occipital gyrus has been reduced, and the white matter magnetic transfer rate and fractional anisotropy adjacent to the visual processing area have been reduced. ${ }^{31,32}$ The occipital cortex of schizophrenia patients showed activity deficits compared with the control group in task-related fMRI studies. ${ }^{33}$ Occipital cortex may be the neurobiological basis of visual processing defects in the early stage of schizophrenia. ${ }^{34}$ In conclusion, the function and structure of occipital lobe may also be related to schizophrenia, which appears in the early course of schizophrenia and persists throughout the course of the disease.

Another area of interest observed in all schizophrenia patients and chronic schizophrenia patients compared with HCs is PreCG, which is an unique region of local functional connectivity alteration. ${ }^{35}$ Evidences confirmed that PreCG plays an important role in social cognition. ${ }^{35}$ Watanuki and his collegues show that the excessive activity of the central anterior gyrus can lead to the emotional processing dysfunction of schizophrenics. ${ }^{36} \mathrm{~A}$ recent study has shown that the severity of clinical symptoms in schizophrenics is related to the disorder of local functional connection in the PreCG. ${ }^{37}$ In a word, the local consistency of PreCG in schizophrenia may be relat-

Table 4. Altered ReHo in chronic schizophrenia patients compared with healthy controls

\begin{tabular}{|c|c|c|c|c|c|c|}
\hline Cluster & Cluster size $\left(\mathrm{mm}^{3}\right)$ & Local extrema $(\mathrm{x}, \mathrm{y}, \mathrm{z})$ & Location & Side & ALE value & Brodman \\
\hline \multicolumn{7}{|c|}{ Decreased ReHo } \\
\hline 1 & 2,032 & $-44,-68,8$ & MOG & $\mathrm{L}$ & 0.0018635334 & 37 \\
\hline 1 & 2,032 & $-44,-74,4$ & MOG & $\mathrm{L}$ & 0.0017753332 & 37 \\
\hline 2 & 1,272 & $50,-66,-10$ & FFG & $\mathrm{R}$ & 0.0017371587 & 37 \\
\hline 2 & 1,272 & $50,-74,-14$ & FFG & $\mathrm{R}$ & 0.0015331637 & 19 \\
\hline 3 & 1,096 & $-56,-10,34$ & PreCG & $\mathrm{L}$ & 0.001846817 & 4 \\
\hline 4 & 864 & $-45,-72,-16$ & Declive & $\mathrm{L}$ & 0.001761289 & - \\
\hline 5 & 808 & $22,-56,62$ & PCUN & $\mathrm{R}$ & 0.0017392386 & 7 \\
\hline 6 & 624 & $-10,46,-20$ & $\mathrm{mFG}$ & $\mathrm{L}$ & 0.0016185765 & 10 \\
\hline 6 & 624 & $-16,48,-16$ & ACC & $\mathrm{L}$ & 0.0014980165 & 32 \\
\hline 7 & 264 & $48,-72,6$ & MOG & $\mathrm{R}$ & 0.0015100014 & 37 \\
\hline
\end{tabular}

MOG: middle occipital gyrus, FFG: fusiform gyrus, PCUN: precuneus, mFG: medial frontal gyrus, ACC: anterior cingulate cortex, R: right, L: left 
ed to the pathophysiological mechanism and clinical symptoms.

We also found increased ReHo in the insula in all schizophrenia patients. Insula is one of the most important parts of the $\mathrm{SN}$, responsible for emotional processing and working memory. ${ }^{38}$ Both Increased ReHo and decreased ReHo were reported in the insula in patients with schizophrenia, ${ }^{11,39}$ while some other studies showed negative results. ${ }^{29,40}$ The reason may be attributed to the heterogeneity of the sample, statistical methods and sample size. A multicenter structural magnetic resonance meta-analysis showed significant thinning of the insula cortex and a significant correlation with drug dose, disease duration and age of onset. ${ }^{41}$ Compared with HCs, functional imaging studies demonstrated reduced local function in the left insula in schizophrenia. ${ }^{11}$ Therefore, insula is unstable in patients with schizophrenia, while the increase of ReHo in the insula of all schizophrenia patients may act a key role in the functional compensation for the systematic structural anomalies.

Interestingly, our study observed inconsistent activation of the frontal lobes. In the all schizophrenia group, we found that the frontal lobe was highly activated, while in the chronic schizophrenia group, the frontal lobe was low activated. Previous studies has demonstrated bilateral frontal gray matter volume is negatively correlated with hallucinations which is one of the main symptoms of schizophrenia. ${ }^{42}$ Many patients commit suicide which is a serious complication of schizophrenia under the influence of hallucinations and delusions. ${ }^{43,44}$ Converging lines of evidence indicate that the phenomenon is directly related to the dysfunction of circuits based on the prefrontal cortex. In conclusion, there are abnormalities of frontal lobe structure and function in schizophrenia, which are related to clinical symptoms and cognitive dysfunction. We speculated that frontal lobe damage is a stable biomarker in schizophrenia. Therefore, the decrease of ReHo in the frontal lobe of patients with chronic schizophrenia may be related to the psychotic symptoms, while the increase of ReHo in the frontal lobe of the first-episode patients may act a pivotal in the compensation of psychotic symptoms of schizophrenia.

In addition to the areas of the brain that were commonly damaged, our study found that in the chronic schizophrenia group decreased ReHo in the PCUN and ACC also was observed, which are mainly located in the DMN. ${ }^{45}$ As we know, $\mathrm{DMN}$ is responsible for the consciousness maintenance, emotional processing, self-introspection, and episodic memory extraction. ${ }^{46}$ Once in disorder, DMN may cause dysfunction, and thus lead to metal illness. ${ }^{46}$ Bluhm et al. ${ }^{47}$ found that the decreased functional connectivity between the cingulate gyrus and multiple default network brain regions in schizophrenia. As a result, the findings of our study are highly credible. Other abnormal brain region like cerebellum also play an im- portant role in chronic schizophrenia. The representative characteristics of damaged cerebellum involve deficits in executive function, linguistic learning, visual spatial processing and regulation of affect. ${ }^{48}$

\section{Limitations}

In our study, some limitations must be pointed out: 1) because there are only three studies on the FES, we have not combined the studies on the FES and can not directly compare the differences between the FES and the chronic patients; 2) individual heterogeneity test cannot be completed by ALE and ALE technology cannot evaluate the significance level of contribution results; 3 ) as suggested by Eickhoff, we used $\mathrm{p}<0.001$ as the threshold for cluster formation and 0.001 for cluster level inference. Most results were significant only before correction for multiple comparisons in false discovery rate (FDR), but our results could not be corrected by FDR, however, in previous simulation work, we determined that uncorrected $\mathrm{p}<0.001$ was empirically equivalent to corrected $\mathrm{p}<0.05$ in our approach, we will use a more stringer correction for multiple comparisons in future study; 4) our meta-analysis samples are all Chinese, which may limit the application of these findings in other populations, further efforts are needed to extend the ReHo method to other patients with schizophrenia; 5) because of the limitation of the study method we could not put other factors (such as PANSS scores, head motion) as covariates into ALE analyses and could not assess the effect of these factors, partially limiting the interpretability and generalizability of the results.

\section{Clinical implications}

Correct understanding of any brain changes in schizophrenia can deepen the understanding of brain development changes related to the occurrence or development of the disease. Based on the meta-analysis of voxels, we found that some brain regions of schizophrenia had local functional connectivity abnormalities. The abnormal spontaneous brain activity may reflect the neuropathological characteristics of schizophrenia, as well as lay a foundation for the establishment of objective diagnostic markers of schizophrenia. Furthermore, our study results give us an enormous assist to determine the course of disease and the role of drugs in different stages of neurodevelopmental disorders in schizophrenia.

\section{Conclusion}

Our meta-analysis showed that the common local abnormal brain areas of the drug-naive patients and chronic patients were MOG, PreCG, frontal lobe and insula, while the brain damage of the chronic patients was more extensive, including PCUN, ACC and cerebellum, which indirectly compared the 
first and chronic patients. Major damaged brain networks include DMN, VN and SN, contributing to our understanding of the progressive pathophysiology of schizophrenia.

\section{Supplementary Materials}

The online-only Data Supplement is available with this article at https://doi.org/10.30773/pi.2021.0062.

\section{Availability of Data and Material}

The datasets generated or analyzed during the study are available from the corresponding author on reasonable request.

\section{Conflicts of Interest}

The authors have no potential conflicts of interest to disclose.

\section{Author Contributions}

Conceptualization: Shiping Xie, Min Zhou. Data curation: Wenwen Xu, Wenying Ma. Formal analysis: Xiaolei Qiu. Funding acquisition: Shiping Xie, Min Zhou. Investigation: all authors. Methodology: Xiaolei Qiu, Rongrong Zhang. Project administration: Shiping Xie, Min Zhou. Resources: Rongrong Zhang, Wei Yan. Software: Xiaolei Qiu, Wenying Ma. Supervision: Shiping Xie. Validation: Shiping Xie, Xiaolei Qiu, Wenwen Xu. Visualization: Xiaolei Qiu. Writing_original draft: Xiaolei Qiu. Writing_review \& editing: Xiaolei Qiu, Rongrong Zhang, Wei Yan, Shiping Xie, Min Zhou.

\section{ORCID iDs}

Xiaolei Qiu

Wenwen Xu

Rongrong Zhang

Wei Yan

Wenying Ma

Shiping Xie

Min Zhou

https://orcid.org/0000-0002-3881-2616 https://orcid.org/0000-0003-4838-7737 https://orcid.org/0000-0002-3117-4869 https://orcid.org/0000-0002-7596-4192 https://orcid.org/0000-0002-3969-3815 https://orcid.org/0000-0002-9947-2440 https://orcid.org/0000-0003-2190-6488

\section{Funding Statement}

This study was partially supported by the General Program of Jiangsu commission of health (H2017051), and the Ministry of Science and Technology of China, National Key R\&D Program of China (2016YFC1306805).

\section{REFERENCES}

1. Kochunov P, Hong LE. Neurodevelopmental and neurodegenerative models of schizophrenia: white matter at the center stage. Schizophr Bull 2014;40:721-728.

2. Birnbaum R, Weinberger DR. Genetic insights into the neurodevelopmental origins of schizophrenia. Nat Rev Neurosci 2017;18:727-740.

3. Fornito A, Zalesky A, Pantelis C, Bullmore ET. Schizophrenia, neuroimaging and connectomics. Neuroimage 2012;62:2296-2314.

4. Gong J, Wang J, Luo X, Chen G, Huang H, Huang R, et al. Abnormalities of intrinsic regional brain activity in first-episode and chronic schizophrenia: a meta-analysis of resting-state functional MRI. J Psychiatry Neurosci 2020;45:55-68.

5. Liu Y, Li M, Chen H, Wei X, Hu G, Yu S, et al. Alterations of regional homogeneity in Parkinson's disease patients with freezing of gait: a resting-state fMRI study. Front Aging Neurosci 2019;11:276.

6. Zang Y, Jiang T, Lu Y, He Y, Tian L. Regional homogeneity approach to fMRI data analysis. Neuroimage 2004;22:394-400.

7. Zuo XN, Xu T, Jiang L, Yang Z, Cao XY, He Y, et al. Toward reliable characterization of functional homogeneity in the human brain: preprocessing, scan duration, imaging resolution and computational space. Neuroimage 2013;65:374-386.
8. Wang S, Zhang Y, Lv L, Wu R, Fan X, Zhao J, et al. Abnormal regional homogeneity as a potential imaging biomarker for adolescent-onset schizophrenia: a resting-state fMRI study and support vector machine analysis. Schizophr Res 2018;192:179-184.

9. Yu R, Hsieh MH, Wang HL, Liu CM, Liu CC, Hwang TJ, et al. Frequency dependent alterations in regional homogeneity of baseline brain activity in schizophrenia. PLoS One 2013;8:e57516.

10. Chen J, Xu Y, Zhang K, Liu Z, Xu C, Shen Y, et al. Comparative study of regional homogeneity in schizophrenia and major depressive disorder. Am J Med Genet B Neuropsychiatr Genet 2013;162B:36-43.

11. Liu C, Xue Z, Palaniyappan L, Zhou L, Liu H, Qi C, et al. Abnormally increased and incoherent resting-state activity is shared between patients with schizophrenia and their unaffected siblings. Schizophr Res 2016;171:158-165.

12. Liu H, Liu Z, Liang M, Hao Y, Tan L, Kuang F, et al. Decreased regional homogeneity in schizophrenia: a resting state functional magnetic resonance imaging study. Neuroreport 2006;17:19-22.

13. Eickhoff SB, Bzdok D, Laird AR, Kurth F, Fox PT. Activation likelihood estimation meta-analysis revisited. Neuroimage 2012;59:2349-2361.

14. Kuhn S, Gallinat J. Resting-state brain activity in schizophrenia and major depression: a quantitative meta-analysis. Schizophr Bull 2013;39:358365.

15. Xiao B, Wang S, Liu J, Meng T, He Y, Luo X. Abnormalities of localized connectivity in schizophrenia patients and their unaffected relatives: a meta-analysis of resting-state functional magnetic resonance imaging studies. Neuropsychiatr Dis Treat 2017;13:467-475.

16. Turkeltaub PE, Eden GF, Jones KM, Zeffiro TA. Meta-analysis of the functional neuroanatomy of single-word reading: method and validation. Neuroimage 2002;16:765-780.

17. Turkeltaub PE, Eickhoff SB, Laird AR, Fox M, Wiener M, Fox P. Minimizing within-experiment and within-group effects in Activation Likelihood Estimation meta-analyses. Hum Brain Mapp 2012;33:1-13.

18. Eickhoff SB, Laird AR, Grefkes C, Wang LE, Zilles K, Fox PT. Coordinate-based activation likelihood estimation meta-analysis of neuroimaging data: a random-effects approach based on empirical estimates of spatial uncertainty. Hum Brain Mapp 2009;30:2907-2926.

19. Yao L, Lui S, Liao Y, Du MY, Hu N, Thomas JA, et al. White matter deficits in first episode schizophrenia: an activation likelihood estimation meta-analysis. Prog Neuropsychopharmacol Biol Psychiatry 2013;45: 100-106.

20. Ran G, Cao X, Chen X. Emotional prediction: an ALE meta-analysis and MACM analysis. Conscious Cogn 2018;58:158-169.

21. Dehghan M, Schmidt-Wilcke T, Pfleiderer B, Eickhoff SB, Petzke F, Harris RE, et al. Coordinate-based (ALE) meta-analysis of brain activation in patients with fibromyalgia. Hum Brain Mapp 2016;37:1749-1758.

22. Shan X, Liao R, Ou Y, Pan P, Ding Y, Liu F, et al. Increased regional homogeneity modulated by metacognitive training predicts therapeutic efficacy in patients with schizophrenia. Eur Arch Psychiatry Clin Neurosci 2021;271:783-798.

23. Zhao X, Yao J, Lv Y, Zhang X, Han C, Chen L, et al. Abnormalities of regional homogeneity and its correlation with clinical symptoms in Naive patients with first-episode schizophrenia. Brain Imaging Behav 2019;13:503-513.

24. Zhang Y, Guo G, Tian Y. Increased temporal dynamics of intrinsic brain activity in sensory and perceptual network of schizophrenia. Front Psychiatry 2019;10:484.

25. Gou N, Liu Z, Palaniyappan L, Li M, Pan Y, Chen X, et al. Effects of DISC1 polymorphisms on resting-state spontaneous neuronal activity in the early-stage of schizophrenia. Front Psychiatry 2018;9:137.

26. Gao B, Wang Y, Liu W, Chen Z, Zhou H, Yang J, et al. Spontaneous activity associated with delusions of schizophrenia in the left medial superior frontal gyrus: a resting-state fMRI study. PLoS One 2015;10: e0133766.

27. Gao S, Lu S, Shi X, Ming Y, Xiao C, Sun J, et al. Distinguishing between treatment-resistant and non-treatment-resistant schizophrenia using 
regional homogeneity. Front Psychiatry 2018;9:282

28. Cui LB, Liu K, Li C, Wang LX, Guo F, Tian P, et al. Putamen-related regional and network functional deficits in first-episode schizophrenia with auditory verbal hallucinations. Schizophr Res 2016;173:13-22.

29. Yan W, Zhang R, Zhou M, Lu S, Li W, Xie S, et al. Relationships between abnormal neural activities and cognitive impairments in patients with drug-naive first-episode schizophrenia. BMC Psychiatry 2020;20:283.

30. Chen J, Xu Y, Zhang K, Liu Z, Xu C, Shen Y, et al. Comparative study of regional homogeneity in schizophrenia and major depressive disorder. Am J Med Genet B Neuropsychiatr Genet 2013;162:36-43.

31. Tohid H, Faizan M, Faizan U. Alterations of the occipital lobe in schizophrenia. Neurosciences (Riyadh) 2015;20:213-224.

32. Palaniyappan L, Al-Radaideh A, Mougin O, Gowland P, Liddle PF. Combined white matter imaging suggests myelination defects in visual processing regions in schizophrenia. Neuropsychopharmacology 2013; 38:1808-1815.

33. Johnston PJ, Stojanov W, Devir H, Schall U. Functional MRI of facial emotion recognition deficits in schizophrenia and their electrophysiological correlates. Eur J Neurosci 2005;22:1221-1232.

34. Smucny J, Tregellas JR. Targeting neuronal dysfunction in schizophrenia with nicotine: Evidence from neurophysiology to neuroimaging. J Psychopharmacol 2017;31:801-811.

35. Jani M, Kasparek T. Emotion recognition and theory of mind in schizophrenia: a meta-analysis of neuroimaging studies. World J Biol Psychiatry 2018;19:S86-S96.

36. Watanuki T, Matsuo K, Egashira K, Nakashima M, Harada K, Nakano $M$, et al. Precentral and inferior prefrontal hypoactivation during facial emotion recognition in patients with schizophrenia: a functional nearinfrared spectroscopy study. Schizophr Res 2016;170:109-114.

37. Aleman A, Tseng HH, Chen SH, Liu CM, Howes O, Huang YL, et al. Facial and prosodic emotion recognition deficits associate with specific clusters of psychotic symptoms in schizophrenia. PLoS One 2013;8: e66571.

38. Uddin LQ. Salience processing and insular cortical function and dys- function. Nat Rev Neurosci 2015;16:55-61.

39. Yu R, Hsieh MH, Wang HLS, Liu CM, Liu CC, Hwang TJ, et al. Frequency dependent alterations in regional homogeneity of baseline brain activity in schizophrenia. PLoS One 2013;8:e57516.

40. Gou NZ, Liu ZN, Palaniyappan L, Li MD, Pan YZ, Chen XD, et al. Effects of DISC1 polymorphisms on resting-state spontaneous neuronal activity in the early-stage of schizophrenia. Front Psychiatry 2018;9:137.

41. van Erp TGM, Walton E, Hibar DP, Schmaal L, Jiang W, Glahn DC, et al. Cortical brain abnormalities in 4474 individuals with schizophrenia and 5098 control subjects via the Enhancing Neuro Imaging Genetics Through Meta Analysis (ENIGMA) Consortium. Biol Psychiatry 2018; 84:644-654.

42. Hodo DW. Psychiatry: Kaplan and Sadock's Synopsis of Psychiatry: Behavioral Sciences/Clinical Psychiatry. JAMA 2003;290:2333-2333.

43. Minzenberg MJ, Lesh TA, Niendam TA, Yoon JH, Rhoades RN, Carter CS. Frontal cortex control dysfunction related to long-term suicide risk in recent-onset schizophrenia. Schizophr Res 2014;157:19-25.

44. Tully LM, Lincoln SH, Liyanage-Don N, Hooker CI. Impaired cognitive control mediates the relationship between cortical thickness of the superior frontal gyrus and role functioning in schizophrenia. Schizophr Res 2014;152:358-364.

45. Woodward ND, Rogers B, Heckers S. Functional resting-state networks are differentially affected in schizophrenia. Schizophr Res 2011;130:8693.

46. Zheng W, Cui B, Han Y, Song H, Li K, He Y, et al. Disrupted regional cerebral blood flow, functional activity and connectivity in Alzheimer's disease: a combined ASL perfusion and resting state fMRI study. Front Neurosci 2019;13:738.

47. Bluhm RL, Miller J, Lanius RA, Osuch EA, Boksman K, Neufeld RW, et al. Spontaneous low-frequency fluctuations in the BOLD signal in schizophrenic patients: anomalies in the default network. Schizophr Bull 2007; 33:1004-1012.

48. Schmahmann JD. The cerebellum and cognition. Neurosci Lett 2019;688: 62-75. 
Supplementary Table 1. Search terms for the systematic literature search

\begin{tabular}{|c|c|}
\hline Database & Search terms \\
\hline PubMed & 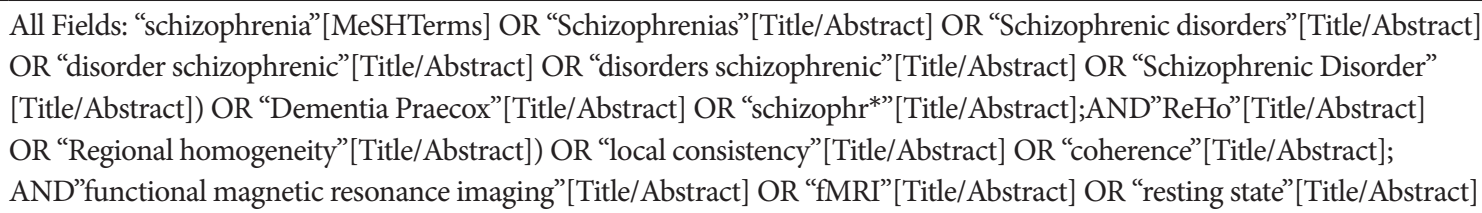 \\
\hline Web of Science & Same as Pubmed \\
\hline Embase & Same as Pubmed \\
\hline Cochranne & Same as Pubmed \\
\hline
\end{tabular}


Category 1: Participants

1. Patients were evaluated prospectively, specific diagnostic criteria were applied, and demographic data were reported.

2. Healthy comparison participants were evaluated prospectively, psychiatric and medical illnesses were excluded.

3. Important variables (e.g., age, sex, illness duration, onset, medication status, comorbidity, severity of illness) were checked either by stratification or statistically.

4. Sample size per group $>10$.

Category 2: Methods for image acquisition and analysis

5. Whole brain analysis was automated with no a priori regional selection.

6. Coordinates reported in a standard space.

7. The imaging technique used was clearly described so that it could be reproduced.

8. Measurements were clearly described so that they could be reproduced.

Category 3: Results and conclusions

9. Statistical parameters for significant and important nonsignificant differences were provided.

10. Conclusions were consistent with the results obtained and the limitations were discussed.

*When criteria were partially met, 0.5 points were awarded 